\title{
Review on application and comparison of metaheuristic techniques to multi-area economic dispatch problem
}

\author{
Jagat Kishore Pattanaik ${ }^{1 *}$, Mousumi Basu ${ }^{1}$ and Deba Prasad Dash ${ }^{2}$
}

\begin{abstract}
This paper presents both application and comparison of the metaheuristic techniques to multi-area economic dispatch (MAED) problem with tie line constraints considering transmission losses, multiple fuels, valve-point loading and prohibited operating zones. The metaheuristic techniques such as differential evolution, evolutionary programming, genetic algorithm and simulated annealing are applied to solve MAED problem. These metaheuristic techniques for MAED problem are evaluated on three different test systems, both small and large, involving varying degree of complexity and the results are compared against each other.
\end{abstract}

Keywords: Multi-area economic dispatch, Tie line constraints, Differential evolution, Evolutionary programming, Genetic algorithm, Simulated annealing

\section{Introduction}

Economic dispatch (ED) is one of the important optimization problems in power system operation. ED allocates the load demand among the committed generators most economically while satisfying the physical and operational constraints in a single area. Generally, the generators are divided into several generation areas interconnected by tie-lines. Multi-area economic dispatch (MAED) is an extension of economic dispatch. MAED determines the generation level and interchange power between areas such that total fuel cost in all areas is minimized while satisfying power balance constraints, generating limits constraints and tie-line capacity constraints.

The economic dispatch problem is frequently solved without considering transmission constraints. However, some researchers have taken transmission capacity constraints into account. Shoults et al. [1] solved economic dispatch problem considering import and export constraints between areas. This study provides a complete formulation of multi-area generation scheduling, and a framework for multi-area studies. Romano

\footnotetext{
* Correspondence: jagat.ju@gmail.com

'Department of Power Engineering, Jadavpur University, Salt Lake City, Kolkata 700098, India

Full list of author information is available at the end of the article
}

et al. [2] presented the Dantzig-Wolfe decomposition principle to the constrained economic dispatch of multi-area systems. An application of linear programming to transmission constrained production cost analysis was proposed in Ref. [3]. Helmick et al. [4] solved multi-area economic dispatch with area control error. Wang and Shahidehpour [5] proposed a decomposition approach for solving multi-area generation scheduling with tie-line constraints using expert systems. Network flow models for solving the multi-area economic dispatch problem with transmission constraints have been proposed by Streiffert [6]. An algorithm for multi-area economic dispatch and calculation of short range margin cost based prices has been presented by Wernerus and Soder [7], where the multi-area economic dispatch problem was solved via Newton-Raphson's method. Yalcinoz and Short [8] solved multi-area economic dispatch problems by using Hopfield neural network approach. Jayabarathi et al. [9] solved multi-area economic dispatch problems with tie line constraints using evolutionary programming. The direct search method for solving economic dispatch problem considering transmission capacity constraints was presented in Ref. [10]. Chen [11] develops a hybrid approach of combining sequential dispatch with a direct search method to deal with the multi-product and multi-area 
electricity market dispatch problem. But these methods did not consider transmission loss.

With the emergence of metaheuristic techniques, attention has been gradually shifted to applications of such technology-based approaches to handle the complexity involved in real world problems. Metaheuristic techniques have been given much attention by many researchers due their ability to seek for the near global optimal solution.

This paper investigates the applicability of the following four metaheuristic techniques in the MAED problem: differential evolution (DE), evolutionary programming (EP), genetic algorithm (GA), and simulated annealing (SA).

Here, three types of MAED problems have been considered. These are A) multi area economic dispatch with quadratic cost function prohibited operating zones and transmission losses (MAEDQCPOZTL) B) multi area economic dispatch with valve point loading (MAEDVPL) C) multi area economic dispatch with valve point loading multiple fuel sources and transmission losses (MAEDVPLMFTL).

The metaheuristic techniques are evaluated against three different test systems for comparison with each other.

\section{Problem formulation}

The objective of MAED is to minimize the total cost of supplying loads to all areas while satisfying power balance constraints, generating limits constraints and tie-line capacity constraints.

Three different types of MAED problems have been considered.

\subsection{MAEDQCPOZTL}

The objective function $F_{t}$, total cost of committed generators of all areas, of MAED problem may be written as

$$
F_{t}=\sum_{i=1}^{\mathrm{N}} \sum_{j=1}^{\mathrm{M}_{\mathrm{i}}} F_{i j}\left(\mathrm{P}_{i j}\right)=\sum_{i=1}^{\mathrm{N}} \sum_{j=1}^{\mathrm{M}_{\mathrm{i}}} a_{i j}+b_{i j} \mathrm{P}_{i j}+c_{i j} \mathrm{P}_{i j}^{2}
$$

where $F_{i j}\left(P_{i j}\right)$ is the cost function of $j$ th generator in area $i$ and is usually expressed as a quadratic polynomial; $a_{i j}$, $b_{i j}$ and $c_{i j}$ are the cost coefficients of $j$ th generator in area $i ; \mathrm{N}$ is the number of areas, $\mathrm{M}_{i}$ is the number of committed generators in area $i ; \mathrm{P}_{i j}$ is the real power output of $j$ th generator in area $i$. The MAED problem minimizes $F_{t}$ subject to the following constraints

\subsubsection{Real power balance constraint}

$$
\sum_{j=1}^{\mathrm{M}_{i}} \mathrm{P}_{i j}=\mathrm{P}_{D i}+\mathrm{P}_{L i}+\sum_{k, k \neq i} \mathrm{~T}_{i k} \quad i \in \mathrm{N}
$$

The transmission loss $P_{L i}$ of area $i$ may be expressed by using B-coefficients as

$$
\mathrm{P}_{L i}=\sum_{l=1}^{\mathrm{M}_{i}} \sum_{j=1}^{\mathrm{M}_{i}} \mathrm{P}_{i j} \mathrm{~B}_{i l j} \mathrm{P}_{i l}+\sum_{j=1}^{\mathrm{M}_{i}} \mathrm{~B}_{0 i j} \mathrm{P}_{i j}+\mathrm{B}_{00 i}
$$

where $\mathrm{P}_{D i}$ is the real power demand of area $i ; \mathrm{T}_{i k}$ is the tie line real power transfer from area $i$ to area $k . \mathrm{T}_{i k}$ is positive when power flows from area $i$ to area $k$ and $\mathrm{T}_{i k}$ is negative when power flows from area $k$ to area $i$.

\subsubsection{Tie line capacity constraints}

The tie line real power transfer $\mathrm{T}_{i k}$ from area $i$ to area $k$ should not exceed the tie line transfer capacity for security consideration.

$$
-\mathrm{T}_{i k}^{\max } \leq \mathrm{T}_{i k} \leq \mathrm{T}_{i k}^{\max }
$$

where $\mathrm{T}_{i k}^{\max }$ is the power flow limit from area $i$ to area $k$ and $-\mathrm{T}_{i k}^{\max }$ is the power flow limit from area $k$ to area $i$.

\subsubsection{Real power generation capacity constraints}

The real power generated by each generator should be within its lower limit $\mathrm{P}_{i j}^{\min }$ and upper limit $\mathrm{P}_{i j}^{\max }$, so that

$$
\mathrm{P}_{i j}^{\min } \leq \mathrm{P}_{i j} \leq \mathrm{P}_{i j}^{\max } \quad i \in \mathrm{N} \text { and } j \in \mathrm{M}_{i}
$$

\subsubsection{Prohibited operating zone}

The prohibited operating zones are the range of power output of a generator where the operation causes undue vibration of the turbine shaft bearing caused by opening or closing of the steam valve. Normally operation is avoided in such regions. The feasible operating zones of unit can be described as follows:

$$
\begin{aligned}
& \mathrm{P}_{i j}^{\min } \leq \mathrm{P}_{i j} \leq \mathrm{P}_{i j, 1}^{l} \\
& \mathrm{P}_{i j, m-1}^{u} \leq \mathrm{P}_{i j} \leq \mathrm{P}_{i j, m}^{l} ; m=2,3, \ldots, n_{i j} \\
& \mathrm{P}_{i j, n_{i j}}^{u} \leq \mathrm{P}_{i j} \leq \mathrm{P}_{i j} \max
\end{aligned}
$$

where $m$ represents the number of prohibited operating zones of $j$ the generator in area $i . \mathrm{P}_{i j, m-1}^{u}$ is the upper limit of $(m-1)$ th prohibited operating zone of $j$ the generator in area $i . \mathrm{P}_{i j, m}^{l}$ is the lower limit of $m$ th prohibited operating zone of $j$ the generator in area $i$. Total number of prohibited operating zone of $j$ the generator in area $i$ is $n_{i j}$.

\subsection{MAEDVPL}

To model the effect of valve-points, a recurring rectified sinusoid contribution is added to the quadratic function [12]. The fuel cost function considering valve-point loadings of the generator is given as 


$$
\begin{aligned}
F t & =\sum_{i=1}^{\mathrm{N}} \sum_{j=1}^{\mathrm{M}_{i}} F_{i j}\left(\mathrm{P}_{i j}\right)=\sum_{i=1}^{\mathrm{N}} \sum_{j=1}^{\mathrm{M}_{i}} a_{i j}+b_{i j} \mathrm{P}_{i j}+c_{i j} \mathrm{P}_{i j}^{2} \\
& +\left|d_{i j} \times \sin \left\{e_{i j} \times\left(\mathrm{P}_{i j}^{\min }-\mathrm{P}_{i j}\right)\right\}\right|
\end{aligned}
$$

where $d_{i j}$ and $e_{i j}$ are cost coefficients of $j$ th generator in area $i$ due to valve-point effect. The objective of MAEDVPL is to minimize $F_{t}$ subject to the constraints given in (2), (4) and (5). Here transmission loss $\left(\mathrm{P}_{L}\right)$ is not considered.

\subsection{MAEDVPLMFTL}

Since generators are practically supplied with multi-fuel sources [13], each generator should be represented with several piecewise quadratic functions superimposed sine terms reflecting the effect of fuel type changes and the generator must identify the most economical fuel to burn. The fuel cost function of the $j$ th generator in area $i$ with $\mathrm{N}_{F}$ fuel types considering valve-point loading is expressed as

$$
\begin{aligned}
F_{i j}\left(\mathrm{P}_{i j}\right) & =a_{i j m}+b_{i j m} \mathrm{P}_{i j}+c_{i j m} \mathrm{P}_{i j}^{2} \\
& +\left|d_{i j m} \times \sin \left\{e_{i j m} \times\left(\mathrm{P}_{i j m}^{\min }-\mathrm{P}_{i j}\right)\right\}\right|
\end{aligned}
$$

if $\mathrm{P}_{i j m}^{\min } \leq \mathrm{P}_{i j} \leq \mathrm{P}_{i j m}^{\max }$ for fuel type $m$ and $m=1,2, \ldots, \mathrm{N}_{F}$ The objective function $F_{t}$ is given by

$$
F_{t}=\sum_{i=1}^{\mathrm{N}} \sum_{j=1}^{\mathrm{M}_{i}} F_{i j}\left(\mathrm{P}_{i j}\right)
$$

The objective function $F_{t}$ is to be minimized subject to the constraints given in (2), (4) and (5).

\section{Determination of generation level of slack generator}

$\mathrm{M}_{i}$ committed generators in area $i$ deliver their power output subject to the power balance constraint (2), tie line capacity constraints (4) and the respective generation capacity constraints (5). Assuming the power loading of first $\left(\mathrm{M}_{i}-1\right)$ generators are known, the power level of the $\mathrm{M}_{i}$ th generator (i.e. the slack generator) is given by

$$
\mathrm{P}_{i \mathrm{M}_{i}}=\mathrm{P}_{D i}+\mathrm{P}_{L i}+\sum_{k, k \neq i} \mathrm{~T}_{i k}-\sum_{j=1}^{\mathrm{M}_{i}-1} \mathrm{P}_{i j}
$$

The transmission loss $\mathrm{P}_{L i}$ is a function of all generator outputs including the slack generator and it is given by

$$
\begin{aligned}
\mathrm{P}_{L i} & =\sum_{l=1}^{\mathrm{M}_{i}-1 \mathrm{M}_{i}-1} \sum_{j=1} \mathrm{P}_{i j} \mathrm{~B}_{i l j} \mathrm{P}_{i l}+2 \mathrm{P}_{i M_{i}}\left(\sum_{j=1}^{\mathrm{M}_{i}-1} \mathrm{~B}_{i \mathrm{M}_{i}} \mathrm{P}_{i j}\right) \\
& +\mathrm{B}_{i \mathrm{M}_{i} \mathrm{M}_{i}} \mathrm{P}_{i \mathrm{M}_{i}}^{2}+\sum_{j=1}^{\mathrm{M}_{i}-1} \mathrm{~B}_{0 i j} \mathrm{P}_{i j}+\mathrm{B}_{0 i \mathrm{M}_{i}} \mathrm{P}_{i \mathrm{M}_{i}}+\mathrm{B}_{00 i}
\end{aligned}
$$

Expanding and rearranging, Eq. (10) becomes

$$
\begin{aligned}
\mathrm{B}_{i \mathrm{M}_{i} \mathrm{M}_{i}} \mathrm{P}_{i \mathrm{M}_{i}}^{2} & +\left(2 \sum_{j=1}^{\mathrm{M}_{i}-1} \mathrm{~B}_{i \mathrm{M}_{i}} \mathrm{P}_{i j}+\mathrm{B}_{0 i \mathrm{M}_{i}}-1\right) \mathrm{P}_{i \mathrm{M}_{i}} \\
& +\left(\mathrm{P}_{D i}+\sum_{k, k \neq i} \mathrm{~T}_{i k}+\sum_{j=1}^{\mathrm{M}_{i}-1 \mathrm{M}_{i}-1} \sum_{l=1}^{\mathrm{P}_{i j}} \mathrm{~B}_{i l j} \mathrm{P}_{i l}\right. \\
& \left.+\sum_{j=1}^{\mathrm{M}_{i}-1} \mathrm{~B}_{0 i j} \mathrm{P}_{i j}-\sum_{j=1}^{\mathrm{M}_{i}-1} \mathrm{P}_{i j}+\mathrm{B}_{00 i}\right)=0
\end{aligned}
$$

The loading of the slack generator (i.e. $\mathrm{M}_{i}$ th) can then be found by solving Eq. (12) using standard algebraic method

\section{Metaheuristic techniques}

Several metaheuristic techniques have evolved in recent past that facilitate to solve optimization problems which were previously difficult or impossible to solve. These techniques include differential evolution, evolutionary programming, genetic algorithm, simulated annealing, etc. Reports of applications of each of these techniques have been widely published. The most important advantage of metaheuristic techniques lies in the fact that they are not limited by restrictive assumptions about the search space like continuity, existence of derivative of objective function, etc.

These methods share some similarities. The DE is introduced first, and followed by EP, GA and SA.

\subsection{Differential evolution}

Differential Evolution (DE) [14] is a type of evolutionary algorithm originally proposed by Price and Storn [15] for optimization problems over a continuous domain. DE is exceptionally simple, significantly faster and robust. The basic idea of $\mathrm{DE}$ is to adapt the search during the evolutionary process. At the start of the evolution, the perturbations are large since parent populations are far away from each other. As the evolutionary process matures, the population converges to a small region and the perturbations adaptively become small. As a result, the evolutionary algorithm performs a global exploratory search during the early stages of the evolutionary process and local exploitation during the mature stage of the search. In DE the fittest of an offspring competes one-to-one with that of corresponding parent which is different from other evolutionary algorithms. This oneto-one competition gives rise to faster convergence rate. 
Price and Storn gave the working principle of DE with simple strategy in [14]. Later on, they suggested ten different strategies of DE [15]. Strategy-7 (DE/rad/1/bin) is the most successful and widely used strategy. The key parameters of control in DE are population size $\left(\mathrm{N}_{\mathrm{P}}\right)$, scaling factor $(F)$ and crossover constant $\left(C_{R}\right)$. The optimization process in $\mathrm{DE}$ is carried out with three basic operations: mutation, crossover and selection. The $\mathrm{DE}$ algorithm is described as follows:

\subsubsection{Initialization}

The initial population of $\mathrm{N}_{\mathrm{P}}$ vectors is randomly selected based on uniform probability distribution for all variables to cover the entire search uniformly. Each individual $X_{i}$ is a vector that contains as many parameters as the problem decision variables $D$. Random values are assigned to each decision parameter in every vector according to:

$$
\mathrm{X}_{i j}^{0} \sim U\left(\mathrm{X}_{j}^{\min }, \mathrm{X}_{j}^{\max }\right)
$$

where $i=1, \ldots ., \mathrm{N}_{\mathrm{P}}$ and $j=1, \ldots, D ; \mathrm{X}_{j}^{\min }$ and $\mathrm{X}_{j}^{\max }$ are the lower and upper bounds of the $j$ th decision variable; $U$ $\left(\mathrm{X}_{j}^{\min }, \mathrm{X}_{j}^{\max }\right)$ denotes a uniform random variable ranging over $\left[\mathrm{X}_{j}^{\min }, \mathrm{X}_{j}^{\max }\right]$. $\mathrm{X}_{i j}^{0}$ is the initial $j$ th variable of $i$ th population. All the vectors should satisfy the constraints. Evaluate the value of the cost function $f\left(\mathrm{X}_{i}^{0}\right)$ of each vector.

\subsubsection{Mutation}

DE generates new parameter vectors by adding the weighted difference vector between two population members to a third member. For each target vector $\mathrm{X}_{i}^{g}$ at $g$ th generation the noisy vector $\mathrm{X}_{i}^{/ g}$ is obtained by

$$
\mathrm{X}_{i}^{/ g}=\mathrm{X}_{a}^{g}+\mathrm{S}_{F}\left(\mathrm{X}_{b}^{g}-\mathrm{X}_{c}^{g}\right), \quad i \in \mathrm{N}_{P}
$$

where $X_{a}^{g a}, X_{b}^{g}$ and $X_{c}^{g}$ are selected randomly from $N_{P}$ vectors at $g$ th generation and $a \neq b \neq c \neq i$. The scaling factor $\left(S_{F}\right)$, in the range $0<S_{F} \leq 1.2$, controls the amount of perturbation added to the parent vector. The noisy vectors should satisfy the constraint.

\subsubsection{Crossover}

Perform crossover for each target vector $X_{i}^{g}$ with its noisy vector $X_{i}^{/ g}$ and create a trial vector $X_{i}^{/ / g}$ such that

$$
X_{i}^{/ / g}=\left\{\begin{array}{ll}
x^{i^{/ g}} & , \text { if } \rho \leq C_{R} \\
X_{i}^{g} & , \text { Otherwise }
\end{array} \quad, \quad i \in \mathrm{N}_{\mathrm{P}}\right.
$$

where $\rho$ is an uniformly distributed random number within $[0,1]$. The crossover constant $\left(C_{R}\right)$, in the range
$0 \leq C_{R} \leq 1$, controls the diversity of the population and aids the algorithm to escape from local optima.

\subsubsection{Selection}

Perform selection for each target vector, $X_{i}^{g}$ by comparing its cost with that of the trial vector, $X_{i}^{/ / g}$. The vector that has lesser cost of the two would survive for the next generation.

$$
\mathrm{X}_{i}^{g+1}=\left\{\begin{array}{ll}
\mathrm{X}_{i}^{/ / g} & , \quad \text { if } \quad f\left(\mathrm{X}_{i}^{/ / g}\right) \leq f\left(\mathrm{X}_{i}^{g}\right) \\
\mathrm{X}_{i}^{g}, & \text { otherwise }
\end{array} \quad, i \in \mathrm{N}_{P}\right.
$$

The process is repeated until the maximum number of generations or no improvement is seen in the best individual after many generations.

\subsection{Evolutionary programming}

Evolutionary Programming (EP) [16] is a technique in the field of evolutionary computation. It seeks the optimal solution by evolving a population of candidate solutions over a number of generations or iterations. During each iteration, a second new population is formed from an existing population through the use of a mutation operator. This operator produces a new solution by perturbing each component of an existing solution by a random amount. The degree of optimality of each of the candidate solutions or individuals is measured by their fitness, which can be defined as a function of the objective function of the problem. Through the use of a competition scheme, the individuals in each population compete with each other. The winning individuals form a resultant population, which is regarded as the next generation. For optimization to occur, the competition scheme must be such that the more optimal solutions have a greater chance of survival than the poorer solutions. Through this the population evolves towards the global optimal point. The algorithm is described as follows:

1) Initialization: The initial population of control variables is selected randomly from the set of uniformly distributed control variables ranging over their upper and lower limits. The fitness score $f_{i}$ is obtained according to the objective function and the environment.

2) Statistics: The maximum fitness $f_{\max }$, minimum fitness $f_{\text {min }}$, the sum of fitness $\Sigma f$, and average fitness $f_{\text {avg }}$ of this generation are calculated.

3) Mutation: Each selected parent, for example $X_{i}$, is mutated and added to its population with the following rule: 
$\mathrm{X}_{i+m, j}=\mathrm{X}_{i j}+N\left(0, \beta\left(\bar{x}_{j}-\underline{x}_{j}\right) \frac{f_{i}}{f_{\max }}\right), \quad j \in n, i \in \mathrm{N}_{\mathrm{P}}$

where $n$ is the number of decision variables in an individual, $N_{P}$ is the population size, $X_{i j}$ denotes the $j$ th element of the $i$ th individual; $N\left(\mu, \sigma^{2}\right)$

represents a Gaussian random variable with mean $\mu$ and variance $\sigma^{2} ; f_{\max }$ is the maximum fitness of the old generation which is obtained in statistics; $\bar{x}_{j}$ and $\underline{x}_{j}$ are respectively maximum and minimum limits of the $j$ th element; and $\beta$ is the mutation scale, $0<\beta \leq$ 1 , that could be adaptively decreased during generations. If any mutated value exceeds its limit, it will be given the limit value. The mutation process allows an individual with larger fitness to produce more offspring for the next generation.

4) Competition: Several individuals $(k)$ which have the best fitness are kept as the parents for the next generation. Other individuals in the combined population of size $\left(2 N_{P}-k\right)$ have to compete with each other to get their chances for the next generation. A weight value $w_{i}$ of the $i$ th individual is calculated by the following competition:

$w_{i}=\sum_{t=1}^{\mathrm{N}_{t}} w_{i, t}$

where $N_{t}$ is the competition number generated randomly; $w_{i, t}$ is either 0 for loss or 1 for win as the $i$ th individual competes with a randomly selected $(r$ th) individual in the combined population. The value of $w_{i, t}$ is given in the following equation:

$w_{i, t}=\left\{\begin{array}{cc}1 & \text { if } f_{i}<f_{r} \\ 0 & \text { Otherwise }\end{array}\right.$

where $f_{r}$ is the fitness of randomly selected $r$ th individuals, and $f_{i}$ is the fitness of the $i$ th individual. When all $2 N_{P}$ individuals, get their competition weights, they will be ranked in a descending order according to their corresponding value $w_{i}$. The first $m$ individuals are selected along with their corresponding fitness $f_{i}$ to be the bases for the next generation. The maximum, minimum and the average fitness and the sum of the fitness of the current generation are then calculated in the statistics.

5) Convergence test: If the convergence condition is not met, the mutation and competition will run again. The maximum generation number can be used for convergence condition. Other criteria, such as the ratio of the average and the maximum fitness of the population is computed and generations are repeated until $\left\{f_{\text {avg }} / f_{\max }\right\} \geq \delta$

where $\delta$ should be very close to 1 , which represents the degree of satisfaction. If the convergence has reached a given accuracy, an optimal solution has been found for an optimization problem.

\subsection{Genetic algorithm}

Genetic algorithm [17] is based on the mechanics of natural selection. An initial population of candidate solutions is created randomly. Each of these candidate solutions is termed as individual. Each individual is assigned a fitness, which measures its quality. During each generation of the evolutionary process, individuals with higher fitness are favored and more probabilities to be selected as parents. After parents are selected for reproduction, they produce children via the processes of crossover and mutation. The individuals formed during reproduction explore different areas of the solution space. These new individuals replace lesser-fit individuals of the existing population.

Due to difficulties of binary representation when dealing with continuous search space with large dimensions, the proposed approach has been implemented using real-coded genetic algorithm (RCGA) [18]. The simulated Binary Crossover (SBX) and polynomial mutation are explained as follows.

\subsubsection{Simulated binary crossover (SBX) operator}

The procedure of computing child populations $c_{1}$ and $c_{2}$ from two parent populations $y_{1}$ and $y_{2}$ under SBX operator as follows:

1. Create a random number $u$ between 0 and 1 .

2. Find a parameter $\gamma$ using a polynomial probability distribution as follows:

$\gamma=\left\{\begin{array}{lrr}(u \alpha)^{1 /\left(\eta_{c}+1\right)}, & \text { if } \quad u \leq \frac{1}{\alpha} \\ (1 /(2-u \alpha))^{1 /\left(\eta_{c}+\right)}, & \text { otherwise }\end{array}\right.$

where $\alpha=2-\beta^{-\left(\eta_{c}+1\right)}$ and

$\beta=1+\frac{2}{y_{2}-y_{1}} \min \left[\left(y_{1}-y_{l}\right),\left(y_{u}-y_{2}\right)\right]$

Here, the parameter $y$ is assumed to vary in $\left[y_{b}, y_{u}\right]$. Here, the parameter $\eta_{c}$ is the distribution index for SBX and can take any non-negative value. A small value of $\eta_{c}$ allows the creation of child populations far away from parents and a large value restricts only nearparent populations to be created as child populations.

3. The intermediate populations are calculated as follows: 
Table 1 Simulation results for test system 1

\begin{tabular}{lllll}
\hline & $\mathrm{DE}$ & $\mathrm{SA}$ & $\mathrm{EP}$ & $\mathrm{RCGA}$ \\
\hline$P_{1,1}(\mathrm{MW})$ & 500.0000 & 500.0000 & 500.0000 & 500.0000 \\
$P_{1,2}(\mathrm{MW})$ & 200.0000 & 200.0000 & 200.0000 & 200.0000 \\
$P_{1,3}(\mathrm{MW})$ & 150.0000 & 150.0000 & 149.9919 & 149.6328 \\
$P_{2,1}(\mathrm{MW})$ & 204.3341 & 204.2157 & 206.4493 & 205.9398 \\
$P_{2,2}(\mathrm{MW})$ & 154.7048 & 155.0575 & 154.8892 & 155.8322 \\
$P_{2,3}(\mathrm{MW})$ & 67.5770 & 67.3516 & 65.2717 & 65.2209 \\
$T_{12}(\mathrm{MW})$ & 82.7731 & 82.7731 & 82.7652 & 82.4135 \\
$P_{L 1}(\mathrm{MW})$ & 9.4269 & 9.4269 & 9.4267 & 9.4193 \\
$P_{L 2}(\mathrm{MW})$ & 4.1890 & 4.1979 & 4.1754 & 4.2064 \\
Cost $(\$ / h)$ & 12255.39 & 12255.39 & 12255.43 & 12256.23 \\
CPU time (second) & 17.6875 & 14.7656 & 21.3281 & 24.2031 \\
\hline
\end{tabular}

$$
\begin{aligned}
& c_{p 1}=0.5\left[\left(y_{1}+y_{2}\right)-\gamma\left(\left|y_{2}-y_{1}\right|\right)\right] \\
& c_{p 2}=0.5\left[\left(y_{1}+y_{2}\right)+\gamma\left(\left|y_{2}-y_{1}\right|\right)\right]
\end{aligned}
$$

Each variable is chosen with a probability $p_{c}$ and the above SBX operator is applied variable-by-variable.

\subsubsection{Polynomial mutation operator}

A polynomial probability distribution is used to create a child population in the vicinity of a parent population under the mutation operator. The following procedure is used:

1. Create a random number $u$ between 0 and 1 .

2. Calculate the parameter $\delta$ as follows: $\delta= \begin{cases}{\left[2 u+(1-2 u)(1-\phi)^{\left(\eta_{m}+1\right)}\right] \frac{1}{\left(\eta_{m}+1\right)}-1,} & \text { if } u \leq 0.5 \\ 1-\left[2(1-u)+2(u-0.5)(1-\phi)^{\left(\eta_{m}+1\right)}\right] \frac{1}{\left(\eta_{m}+1\right)}, & \text { otherwise }\end{cases}$

where $\phi=\frac{\min \left[\left(c_{p}-y_{l}\right),\left(y_{u}-c_{p}\right)\right]}{\left(y_{u}-y_{l}\right)}$

The parameter $\eta_{m}$ is the distribution index for mutation and takes any non-negative value.

3. Calculate the mutated child as follows:

$c_{1}=c_{p 1}+\delta\left(y_{u}-y_{l}\right)$

$c_{2}=c_{p 2}+\delta\left(y_{u}-y_{l}\right)$

The perturbance in the population can be adjusted by varying $\eta_{m}$ and $p_{m}$ with generations as given below:

$\eta_{m}=\eta_{m \min }+g e n$

$p_{m}=\frac{1}{n}+\frac{g e n}{g e n_{\max }}\left(1-\frac{1}{n}\right)$

where $\eta_{m \text { min }}$ is the user defined minimum value for $\eta_{m}, p_{m}$ is the probability of mutation, and $n$ is the number of decision variables

\subsection{Simulated annealing}

Simulated annealing [19] is a powerful optimization technique which exploits the resemblance between a minimization process and the cooling of molten metal. The physical annealing process is simulated in the simulated

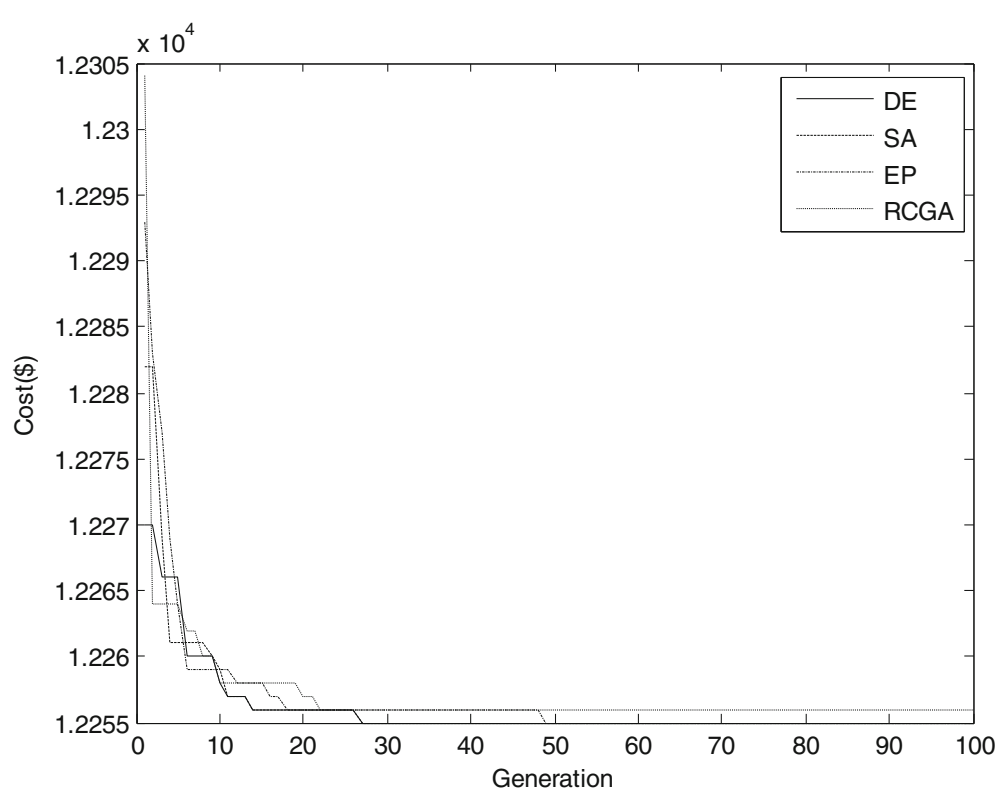

Fig. 1 Cost convergence characteristic of test system 1 
Table 2 Simulation results for test system 2

\begin{tabular}{|c|c|c|c|c|c|c|c|c|}
\hline \multirow[t]{2}{*}{ Power (MW) } & \multicolumn{2}{|l|}{ DE } & \multicolumn{2}{|l|}{ SA } & \multicolumn{2}{|l|}{ EP } & \multicolumn{2}{|l|}{ RCGA } \\
\hline & & Fuel & & Fuel & & Fuel & & Fuel \\
\hline$P_{1,1}(\mathrm{MW})$ & 225.9431 & 2 & 228.1730 & 2 & 223.8491 & 2 & 239.0958 & 2 \\
\hline$P_{1,2}(\mathrm{MW})$ & 211.1594 & 1 & 213.3402 & 1 & 209.5759 & 1 & 216.1166 & 1 \\
\hline$P_{1,3}(\mathrm{MW})$ & 489.9216 & 2 & 482.8722 & 2 & 496.0680 & 2 & 484.1506 & 2 \\
\hline$P_{1,4}(\mathrm{MW})$ & 240.6232 & 3 & 242.6425 & 3 & 237.9954 & 3 & 240.6228 & 3 \\
\hline$P_{2,1}(\mathrm{MW})$ & 254.0397 & 1 & 253.5059 & 1 & 259.4299 & 1 & 259.6639 & 1 \\
\hline$P_{2,2}(\mathrm{MW})$ & 235.4927 & 3 & 236.5760 & 3 & 228.9422 & 3 & 219.9107 & 3 \\
\hline$P_{2,3}(\mathrm{MW})$ & 263.8837 & 1 & 266.6356 & 1 & 264.1133 & 1 & 254.5140 & 1 \\
\hline$P_{3,1}(\mathrm{MW})$ & 237.0006 & 3 & 234.3130 & 3 & 238.2280 & 3 & 231.3565 & 3 \\
\hline$P_{3,2}(\mathrm{MW})$ & 328.7373 & 1 & 325.9516 & 1 & 331.2982 & 1 & 341.9624 & 1 \\
\hline$P_{3,3}(\mathrm{MW})$ & 248.8607 & 1 & 251.4034 & 1 & 246.6025 & 1 & 248.2782 & 1 \\
\hline$T_{21}(\mathrm{MW})$ & 99.8288 & & 100 & & 100 & & 93.1700 & \\
\hline$T_{31}(\mathrm{MW})$ & 99.7334 & & 99.8797 & & 100 & & 93.8739 & \\
\hline$T_{32}(\mathrm{MW})$ & 31.2615 & & 28.1853 & & 32.5231 & & 43.7824 & \\
\hline$P_{L 1}(\mathrm{MW})$ & 17.2095 & & 16.9000 & & 17.4884 & & 17.0297 & \\
\hline$P_{L 2}(\mathrm{MW})$ & 9.8488 & & 9.9028 & & 10.0085 & & 9.7010 & \\
\hline$P_{L 3}(\mathrm{MW})$ & 8.6037 & & 8.6030 & & 8.6056 & & 8.9408 & \\
\hline Cost $(\$ / h)$ & 653.9995 & & 654.0916 & & 655.1716 & & 657.3325 & \\
\hline CPU time (second) & 95.0351 & & 10.0156 & & 108.0625 & & 133.8438 & \\
\hline
\end{tabular}

annealing (SA) technique for the determination of global or near-global optimum solutions for optimization problems. In this algorithm, a parameter $T_{0}$, called temperature, is defined. Starting from a high temperature, a molten metal is cooled slowly until it is solidified at a low temperature. The iteration number in the SA technique is analogous to the temperature level. During each iteration, a candidate solution is generated. If this solution is a better solution, it will be accepted and used to generate yet another candidate solution. If it is a deteriorated solution, the solution will be accepted when its probability of acceptance $\operatorname{Pr}(\Delta)$ as given by Eq. (26) is greater than a randomly generated number between 0 and 1 :

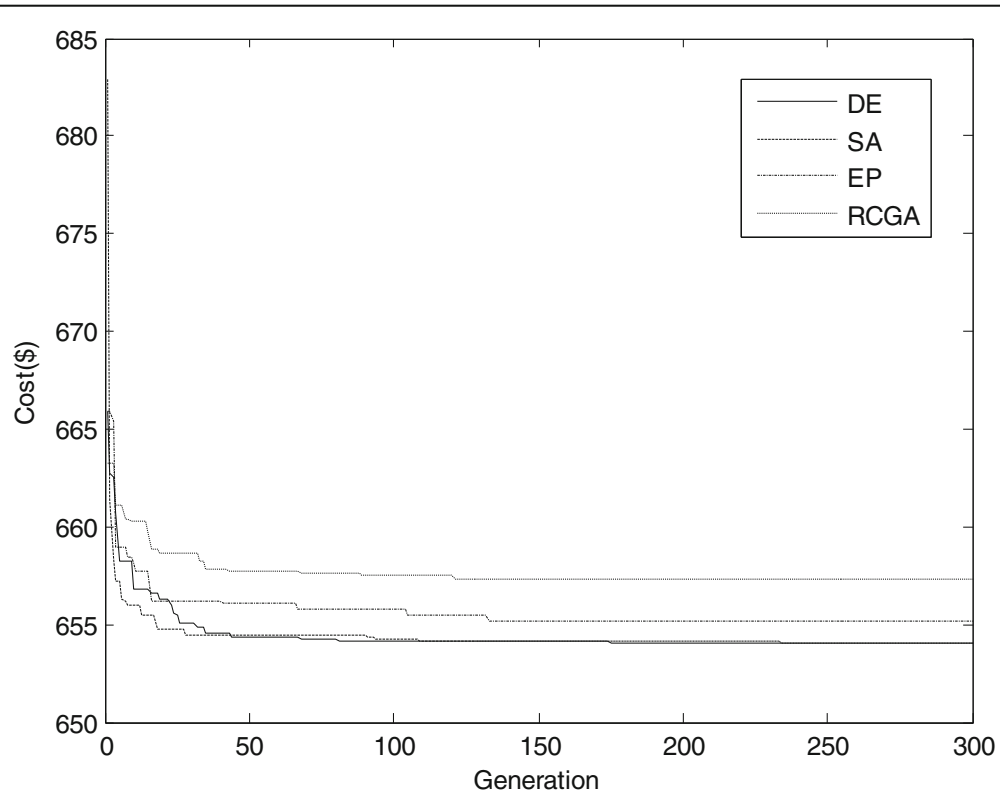

Fig. 2 Cost convergence characteristic of test system 2 
Table 3 Simulation results for test system 3

\begin{tabular}{|c|c|c|c|c|c|c|c|c|c|}
\hline Power (MW) & $\mathrm{DE}$ & SA & EP & RCGA & Power (MW) & DE & SA & EP & RCGA \\
\hline$P_{1,1}$ & 111.5448 & 110.9120 & 107.6644 & 95.7552 & $P_{3,4}$ & 523.4073 & 523.3366 & 525.7752 & 518.1120 \\
\hline$P_{1,2}$ & 111.7092 & 111.8740 & 112.0673 & 88.5828 & $P_{3,5}$ & 523.7703 & 525.5247 & 531.2092 & 538.1994 \\
\hline$P_{1,3}$ & 98.2429 & 110.2589 & 91.8132 & 97.6063 & $P_{3,6}$ & 523.5424 & 523.2794 & 513.5659 & 527.4775 \\
\hline$P_{1,4}$ & 179.8834 & 179.7351 & 175.3171 & 126.4966 & $P_{3,7}$ & 10.1621 & 10.0002 & 11.3612 & 24.4133 \\
\hline$P_{1,5}$ & 95.9500 & 88.8739 & 92.4242 & 71.0127 & $P_{3,8}$ & 10.1326 & 10.0006 & 10.0000 & 28.9856 \\
\hline$P_{1,6}$ & 139.3533 & 68.0000 & 112.5634 & 116.3866 & $P_{3,9}$ & 10.6366 & 10.0006 & 10.0000 & 28.8571 \\
\hline$P_{1,7}$ & 259.3395 & 184.9322 & 257.5370 & 244.5857 & $P_{3,10}$ & 88.1189 & 93.2065 & 78.3523 & 87.9016 \\
\hline$P_{1,8}$ & 285.3569 & 285.0432 & 297.3619 & 210.6920 & $P_{4,1}$ & 161.2220 & 190.0000 & 162.4480 & 159.7482 \\
\hline$P_{1,9}$ & 284.9627 & 284.6015 & 285.2035 & 236.1685 & $P_{4,2}$ & 189.5668 & 189.9990 & 166.3508 & 153.6255 \\
\hline$P_{1,10}$ & 130.2217 & 130.0008 & 134.5862 & 130.1286 & $P_{4,3}$ & 189.9240 & 159.7546 & 190.0000 & 160.4706 \\
\hline$P_{2,1}$ & 243.6005 & 168.6194 & 162.4313 & 367.4862 & $P_{4,4}$ & 165.6621 & 165.6736 & 178.4541 & 169.9359 \\
\hline$P_{2,2}$ & 95.3890 & 318.3986 & 217.8387 & 297.9501 & $P_{4,5}$ & 165.4321 & 164.8248 & 168.0752 & 168.5220 \\
\hline$P_{2,3}$ & 214.5171 & 304.5197 & 125.0000 & 394.9246 & $P_{4,6}$ & 164.9868 & 196.1794 & 174.4529 & 172.2638 \\
\hline$P_{2,4}$ & 394.0808 & 394.2792 & 384.0187 & 370.3473 & $P_{4,7}$ & 109.8137 & 89.1143 & 77.3875 & 91.2423 \\
\hline$P_{2,5}$ & 394.2481 & 469.0618 & 397.6902 & 455.7123 & $P_{4,8}$ & 109.7935 & 89.1147 & 90.1059 & 86.4778 \\
\hline$P_{2,6}$ & 394.4360 & 304.5195 & 407.4993 & 393.9673 & $P_{4,9}$ & 90.1543 & 104.7206 & 109.5654 & 88.3627 \\
\hline$P_{2,7}$ & 489.9552 & 489.2801 & 500.0000 & 424.1994 & $P_{4,10}$ & 459.1140 & 458.7992 & 549.0335 & 279.2691 \\
\hline$P_{2,8}$ & 488.8885 & 489.2803 & 480.8874 & 484.5498 & $T_{12}$ & 172.0652 & 192.6532 & 200 & -71.7855 \\
\hline$P_{2,9}$ & 511.4713 & 511.2790 & 524.8487 & 528.4148 & $T_{31}$ & -36.3060 & 160.6028 & 17.5885 & 161.9336 \\
\hline$P_{2,10}$ & 511.4125 & 511.2805 & 499.7857 & 511.3403 & $T_{32}$ & 191.1128 & -46.9736 & 200 & 95.2833 \\
\hline$P_{3,1}$ & 523.2896 & 524.8208 & 523.4522 & 525.4497 & $T_{41}$ & 86.8070 & 52.8188 & 90.8733 & -76.1340 \\
\hline$P_{3,2}$ & 523.2950 & 523.2802 & 526.5051 & 510.7391 & $T_{42}$ & 98.8231 & 93.8021 & 100 & -52.3900 \\
\hline$P_{3,3}$ & 523.4129 & 433.6204 & 537.3675 & 533.6399 & $T_{43}$ & 45.0391 & 86.5590 & 100 & 83.4418 \\
\hline \multicolumn{6}{|c|}{ Total cost $(\$ / h)$} & 121794.8 & 123337.1 & 123591.9 & 128046.5 \\
\hline \multicolumn{6}{|c|}{ CPU time (second) } & 134.8125 & 29.2813 & 144.5000 & 160.5313 \\
\hline
\end{tabular}

$$
\operatorname{Pr}(\Delta)=1 /\left(1+\exp \left(\Delta / \mathrm{T}_{v}\right)\right)
$$

where $\Delta$ is the amount of deterioration between the new and the current solutions and $T_{\nu}$ is the temperature at which the new solution is generated. Accepting deteriorated solutions in the above manner enables the SA solution to 'jump' out of the local optimum solution points and to seek the global optimum solution. In forming the new solution the current solution is perturbed [20] according to the Gaussian probability distribution function (GPDF). The mean of the GPDF is taken to be the current solution, and its standard deviation is given by the product of the temperature and a scaling factor $\sigma$. The value of $\sigma$ is less than one, and together with the value of temperature, it governs the size of the neighborhood space of the current solution and hence the amount of perturbation. The amount of perturbation is dependent upon the temperature when $\sigma$ is kept at a constant value. In each iteration, the procedure for generating and testing the candidate solution is repeated for a specified number of trials so that thermal equilibrium is reached for each temperature. The last accepted candidate solution is then taken as the starting solution for the generation of candidate solutions in the next iteration. Simulated annealing with a slow cooling schedule usually has larger capacity to find the optimal solution than that of a fast cooling schedule. The reduction of the temperature in successive iterations is governed by the following geometric function [19]

$$
\mathrm{T}_{v}=r^{(v-1)} \mathrm{T}_{0}
$$

where $v$ is the iteration number and $r$ is temperature reduction factor. $T_{0}$ is the initial temperature, the value of which can be set arbitrarily or estimated using the method described in reference [20]. The iterative process is terminated when there is no significant improvement in the solution after a prespecified number of iterations. It can also be terminated when the maximum number of iterations is reached.

\subsection{Simulation results}

A comparative study is performed for the four metaheuristic techniques by solving the MAED problem for three different test systems. All metaheuristic techniques 


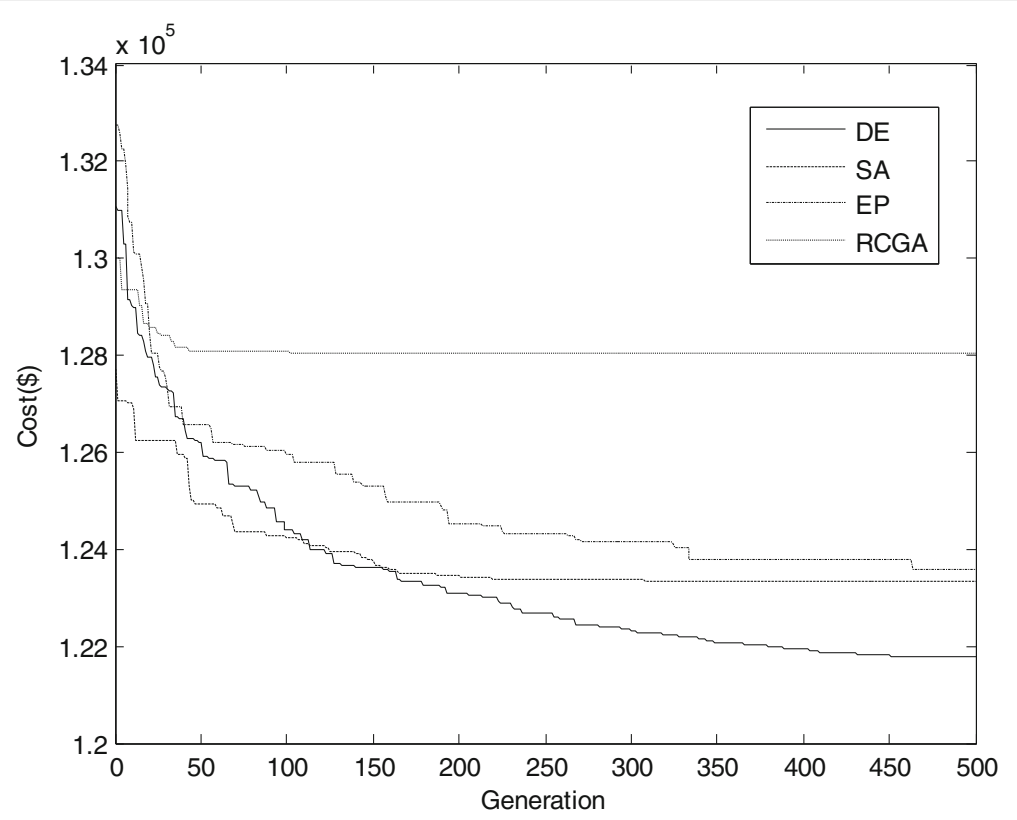

Fig. 3 Cost convergence characteristic of test system 3

for the MAED problems are implemented by using MATLAB 7.0 on a PC (Pentium-IV, $80 \mathrm{~GB}, 3.0 \mathrm{GHz}$ ).

The initial temperature $\left(T_{0}\right)$ of SA algorithm has been determined by using the procedures described in [20]. As per guideline [19], the value of $r$ lies in the range from 0.80 to 0.99. For seeking the optimal solution, the value of $r$ is required to be set close to 0.99 so that a slow cooling process is simulated. The appropriate setting of $r$ is set by experimenting with its value in the range from 0.95 to 0.99 , and this value is found to be 0.98 . Number of trials at each temperature has been taken 30. In this paper, iterative process is terminated when the maximum number of iterations is reached.

\subsubsection{Test system 1}

This system consists of two areas. Each area consists of three generators with prohibited operating zones. Transmission loss is considered here. The generator data has modified from [21]. The generator data and B-coefficients are given in the Appendix 1. The percentage of the total load demand in area 1 is $60 \%$ and $40 \%$ in area 2 . The total load demand is $1263 \mathrm{MW}$ and power flow limit of the system is $100 \mathrm{MW}$.

The problem is solved by using DE, EP, RCGA, and SA. In case of $\mathrm{DE}$, the population size, scaling factor, and crossover rate have been selected as $100,0.75$, and 1.0 respectively for the test system under consideration. The population size and scaling factor have been selected as 100 , and 0.1 respectively in case of EP. In case of RCGA, the population size, crossover and mutation probabilities have been selected as 100, 0.9 and 0.2 respectively.

Maximum number of generations has been selected 100 for all the four metaheuristic techniques discussed in this paper.
Results obtained from the four metaheuristic techniques i.e. DE, EP, RCGA, and SA have been summarized in Table 1. Figure 1 gives the comparison of convergence of minimum total cost obtained by DE, EP, RCGA, and SA.

\subsubsection{Test system 2}

This system comprises ten generators with valve-point loading and multi-fuel sources having three fuel options. Transmission loss is considered here. The generator data has been taken from [13]. The total load demand is 2700 MW. The ten generators are divided into three areas. Area 1 consists of the first four units; area 2 includes the next three units and area 3 includes the last three units. The load demand in area 1 is assumed as $50 \%$ of the total demand. The load demand in area 2 is assumed as $25 \%$ and in area 3 is taken as $25 \%$ of the total demand. The power flow limit from area 1 to area 2 or from area 2 to area 1 is $100 \mathrm{MW}$. The power flow limit from area 1 to area 3 or from area 3 to area 1 is $100 \mathrm{MW}$. Also the power flow limit from area 2 to area 3 or from area 3 to area 2 is 100 MW. The B-coefficients are given in the Appendix 2.

The problem is solved by using four metaheuristic techniques i.e. DE, EP, RCGA, and SA.

In case of $\mathrm{DE}$, the population size, scaling factor, and crossover rate have been selected as $200,0.75$, and 1.0 respectively for the test system under consideration. The population size and scaling factor have been selected as 100, and 0.1 respectively in case of EP. In case of RCGA, the population size, crossover and mutation probabilities have been selected as 100, 0.9 and 0.2 respectively. Maximum number of generations has been selected 300 for DE, EP, RCGA, and SA. 
Results obtained from DE, EP, RCGA and RCGA have been presented in Table 2 . The cost convergence characteristic of this test system obtained from DE, EP, RCGA and SA is shown in Fig. 2.

\subsubsection{Test system 3}

This system comprises forty generators with valve-point loading. The generator data has been taken from [22]. The total load demand is $10500 \mathrm{MW}$. The forty generators are divided into four areas. Area 1 includes first ten units and 15\% of the total load demand. Area 2 has second ten generators and $40 \%$ of the total load demand. Area 3 consists of third ten generators and $30 \%$ of the total load demand. Area four includes last ten generators and $15 \%$ of the total load demand. The power flow limit from area 1 to area 2 or from area 2 to area 1 is $200 \mathrm{MW}$. The power flow limit from area 1 to area 3 or from area 3 to area 1 is $200 \mathrm{MW}$. The power flow limit from area 2 to area 3 or from area 3 to area 2 is $200 \mathrm{MW}$. The power flow limit from area 4 to area 1 or from area 1 to area 4 is $100 \mathrm{MW}$. The power flow limit from area 4 to area 2 or from area 2 to area 4 is $100 \mathrm{MW}$. The power flow limit from area 4 to area 3 or from area 3 to area 4 is $100 \mathrm{MW}$.

Transmission loss is neglected here.

Four metaheuristic techniques i.e. DE, EP, RCGA, and SA have been used to solve the problem.

The population size, scaling factor, and crossover rate have been been selected as 400, 0.75 and 1.0 respectively in case of DE. In EP, the population size and scaling factor have been selected 200 and 0.1 respectively. In case of RCGA, the population size, crossover and mutation probabilities have been selected as 200, 0.9 and 0.2 respectively. Maximum number of generations has been selected 500 for DE, EP, RCGA and SA.

Results obtained from DE, EP, RCGA and SA have been depicted in Table 3 . The cost convergence characteristic of this test system obtained from DE, EP, RCGA and SA is shown in Fig. 3.

From Tables 1, 2 and 3, it can be inferred that, the lowest minimum total cost amongst the four is achieved by DE, followed by SA. Minimum total cost obtained by EP is more than DE and SA. RCGA is the worst performer. The CPU time requirement is least in case of SA and highest in the case of RCGA amongst the four metaheuristic techniques discussed in the paper.

\section{Conclusion}

In this paper, a comparison analysis has been done for the four metaheuristic techniques viz., differential evolution, evolutionary programming, real coded genetic algorithm and simulated annealing technique for multi-area economic dispatch problem considering transmission losses, multiple fuels, valve-point loading and prohibited operating zones with respect to minimum cost and CPU time. Differential evolution achieves the lowest minimum cost and SA requires least CPU time amongst the four metaheuristic techniques.

\section{Appendix 1}

Table 4 Data for 2 area system

\begin{tabular}{|c|c|c|c|c|c|c|}
\hline Generator ij & $\begin{array}{l}a_{i j} \\
\$ / h\end{array}$ & $\begin{array}{l}b_{i j} \\
\$ / M W h\end{array}$ & $\begin{array}{l}c_{i j} \\
\$ /(M W)^{2} h\end{array}$ & $\begin{array}{l}\mathrm{P}_{i j}^{\min } \\
\mathrm{MW}\end{array}$ & $\begin{array}{l}P_{i j}^{\max } \\
\mathrm{MW}\end{array}$ & $\begin{array}{l}\text { Prohibited zones } \\
\text { MW }\end{array}$ \\
\hline$G_{1,1}$ & 550 & 8.10 & 0.00028 & 100 & 500 & {$\left[\begin{array}{lll}210 & 240\end{array}\right]\left[\begin{array}{lll}350 & 380\end{array}\right]$} \\
\hline$G_{1,2}$ & 350 & 7.50 & 0.00056 & 50 & 200 & {$\left[\begin{array}{ll}90 & 110]\end{array}\left[\begin{array}{ll}140 & 160\end{array}\right]\right.$} \\
\hline$G_{1,3}$ & 310 & 8.10 & 0.00056 & 50 & 150 & [80 90] [110 120] \\
\hline 6 & 240 & 7.74 & 0.00324 & 80 & 300 & {$\left[\begin{array}{lll}150 & 170\end{array}\right]\left[\begin{array}{ll}210 & 240\end{array}\right]$} \\
\hline$G_{2,2}$ & 200 & 8.00 & 0.00254 & 50 & 200 & {$\left[\begin{array}{ll}90 & 110\end{array}\right]\left[\begin{array}{ll}140 & 150\end{array}\right]$} \\
\hline$G_{2,3}$ & 126 & 8.60 & 0.00284 & 50 & 120 & [75 85] [100 105] \\
\hline
\end{tabular}

The transmission loss formula coefficients of two-area system are:

$$
\begin{aligned}
& \mathrm{B}_{1}=\left[\begin{array}{ccc}
17 & 12 & 7 \\
12 & 14 & 9 \\
7 & 9 & 31
\end{array}\right] \quad \mathrm{X} 10^{-6} \\
& \mathrm{~B}_{01}=\left[\begin{array}{lll}
-0.3908 & -0.1297 & 0.7047
\end{array}\right] \quad \mathrm{X} 10^{-3} \\
& \mathrm{~B}_{001}=0.045 \\
& \mathrm{~B}_{2}=\left[\begin{array}{ccc}
24 & -6 & -8 \\
-6 & 129 & -2 \\
-8 & -2 & 150
\end{array}\right] \quad \mathrm{X} 10^{-6} \\
& \mathrm{~B}_{02}=\left[\begin{array}{lll}
0.0591 & 0.2161 & -0.6635
\end{array}\right] \quad \mathrm{X} 10^{-3} \\
& \mathrm{~B}_{002}=0.056
\end{aligned}
$$

\section{Appendix 2}

The transmission loss formula coefficients of three-area system are:

$$
\begin{aligned}
& \mathrm{B}_{1}=\left[\begin{array}{cccc}
8.70 & 0.43 & -4.61 & 0.36 \\
0.43 & 8.30 & -0.97 & 0.22 \\
-4.61 & -0.97 & 9.00 & -2.00 \\
0.36 & 0.22 & -2.00 & 5.30
\end{array}\right] \mathrm{X} 0^{-5} \\
& \mathrm{~B}_{01}=\left[\begin{array}{llll}
-0.3908 & -0.1297 & 0.7047 & 0.0591
\end{array}\right] \quad \times 10^{-3} \\
& \mathrm{~B}_{001}=0.056 \\
& \mathrm{~B}_{2}=\left[\begin{array}{ccc}
8.60 & -0.80 & 0.37 \\
-0.80 & 9.08 & -4.90 \\
0.37 & -4.90 & 8.24
\end{array}\right] \mathrm{X} 10^{-5} \\
& \mathrm{~B}_{02}=\left[\begin{array}{lll}
0.2161 & -0.6635 & 0.5034
\end{array}\right] \mathrm{X} 10^{-3} \\
& \mathrm{~B}_{002}=0.045
\end{aligned}
$$




$$
\begin{aligned}
& \mathrm{B}_{3}=\left[\begin{array}{ccc}
1.20 & -0.96 & 0.56 \\
-0.96 & 4.93 & -0.30 \\
0.56 & -0.30 & 5.99
\end{array}\right] \quad \times 10^{-5} \\
& \mathrm{~B}_{03}=\left[\begin{array}{llll}
-0.3216 & 0.4635 & 0.3503
\end{array}\right] \quad{\mathrm{X} 10^{-3}}^{-3} \\
& \mathrm{~B}_{003}=0.055
\end{aligned}
$$

\section{Funding}

There is NO Funding Information available for this manuscript.

\section{Authors' contributions}

JKP makes substantial contributions to conception, design and acquisition of data analysis and interpretation of data. JKP drafted the article and revising it thoroughly for preparation of the manuscript for the esteemed journal. Also he did the simulation part by using different test data for three different test systems. As a corresponding author he takes the primary responsibility for communication of the journal during the manuscript submission, peer review, publication process, and typically ensures that all the journal's administrative requirements, such as providing details of authorship. JKP will be available throughout the submission and peer review process to respond to editorial queries in a timely manner. Also he will be available after publication to respond to critiques of the work and cooperate with any requests from the journal for data or additional information should be answered about the paper arise after publication. JKP also agrees to be accountable for all aspects of the work in ensuring that questions related to the accuracy or integrity of any part of the work are appropriately investigated and resolved. MB participated in the peer review process of the manuscript and involved in the test data preparation. She reviewed the manuscript thoroughly. DPD participated in the peer review process of the manuscript and to compare the performance of the proposed method with that of other evolutionary methods. All authors read and approved the final manuscript.

\section{Authors' information}

Jagat Kishore Pattanaik received the Master's degree in Electrical Engineering in 2011 from Jadavpur University, Kolkata, India. He is currently working towards the Ph.D degree in the Department of Power Engineering, Jadavpur University. His research interest is Soft computing application in Power system Engineering.

Dr. Mousumi Basu received the Ph.D. degree from Jadavpur University, Kolkata, India. She is currently working as an Associate Professor in the Department of Power Engineering, Jadavpur University. His research interest is Power system Optimization and Soft computing technique. Dr. Deba Prasad Dash received the Ph.D. degree from Jadavpur University, Kolkata, India. He is currently working as a Professor in the Department of Electrical Engineering, Orissa Engineering College, Bhubaneswar, Odisha, India. His research interests are Power system Stability, Power system protection \& Soft computing technique.

\section{Competing interests}

We have declared that we have NO significant competing financial, professional or personal interests that might have influenced the performance or presentation of the work described in the manuscript.

\section{Author details}

'Department of Power Engineering, Jadavpur University, Salt Lake City, Kolkata 700098, India. ${ }^{2}$ Electrical Engineering Department, Orissa Engineering College, Bhubaneswar, India.

Received: 3 July 2016 Accepted: 18 April 2017

Published online: 03 May 2017

\section{References}

1. Shoults, R. R., Chang, S. K., Helmick, S., \& Grady, W. M. (1980). A practical approach to unit commitment, economic dispatch and savings allocation for multiple-area pool operation with import/export constraints. IEEE Trans Power Apparatus Syst., 99(2), 625-635.
2. Romano, R., Quintana, V. H., Lopez, R., \& Valadez, V. (1981). Constrained economic dispatch of multi-area systems using the Dantzig-Wolfe decomposition principle. IEEE Trans. Power Apparatus Syst., 100(4), 2127-2137.

3. Desell, A. L., McClelland, E. C., Tammar, K., \& Van Horne, P. R. (1984). Transmission constrained production cost analysis in power system planning. IEEE Trans Power Apparatus Syst., 103(8), 2192-2198.

4. Helmick, S. D., \& Shoults, R. R. (1985). A practical approach to an interim multi-area economic dispatch using limited computer resources. IEEE Trans Power Apparatus Syst., 104(6), 1400-1404.

5. Wang, C., \& Shahidehpour, S. M. (1992). A decomposition approach to nonlinear multi area generation scheduling with tie-line constraints using expert systems. IEEE Transactions on Power Systems, 7(4), 1409-1418.

6. Streiffert, D. (1995). Multi-area economic dispatch with tie line constraints. IEEE Transactions on Power Systems, 10(4), 1946-1951.

7. Wernerus, J, Soder, L (1995) Area price based multi-area economic dispatch with tie line losses and constraints. In: IEEE/KTH Stockholm power tech conference (pp. 710-715). Sweden

8. Yalcinoz, T., \& Short, M. J. (1998). Neural networks approach for solving economic dispatch problem with transmission capacity constraints. IEEE Transactions on Power Systems, 13(2), 307-313.

9. Jayabarathi, T., Sadasivam, G., \& Ramachandran, V. (2000). Evolutionary programming based multi-area economic dispatch with tie line constraints. Electric Machine and Power System, 28, 1165-1176.

10. Chen, C. L., \& Chen, N. (2001). Direct Search Method for solving Economic Dispatch Problem Considering Transmission Capacity Constraints. IEEE Transactions on Power Systems, 16(4), 764-769.

11. Chen, C. L. (2005). Optimal generation and reserve dispatch in a multi-area competitive market using a hybrid direct search method. Energy Conversion and Management, 46, 2856-2872.

12. Walter, D. C., \& Sheble, G. B. (1993). Genetic algorithm solution of economic dispatch with valve point loading. IEEE Transactions on Power Systems, 8, 1325-1332.

13. Chiang, C.-L. (2005). Improved genetic algorithm for power economic dispatch of units with valve-point effects and multiple fuels. IEEE Transactions on Power Systems, 20(4), 1690-1699.

14. Storn, R., \& Price, K. V. (1997). Differential evolution- a simple and efficient heuristic for global optimization over continuous spaces. Journal of Global Optimization, 11(4), 341-359.

15. Price, K. V., Storn, R., \& Lampinen, J. (2005). Differential Evolution: A Practical Approach to Global Optimization. Berlin: Springer.

16. Yang, H. T., Yang, P. C., \& Huang, C. L. (1996). Evolutionary Programming based economic dispatch for units with non-smooth fuel cost functions. IEEE transactions on Power Systems, 11(1), 112-118.

17. Goldberg, D. (1989). Genetic Algorithms in Search, Optimization \& Machine Learning. Reading: Addison-Wesley Publishing Company, Inc.

18. Deb, K., \& Agrawal, R. B. (1995). Simulated binary crossover for continuous search space. Complex Systems, 9(2), 115-148.

19. Kirkpatrick, S., Gelatt, C., \& Vecchi, M. (1983). Optimization by simulated annealing. Science, 22, 671-680.

20. Wong, K. P., \& Fung, C. C. (1993). Simulated annealing based economic dispatch algorithm. IEE Proceedings Generation Transmission and Distribution, 140(6), 509-515.

21. Gaing, Z.-L. (2003). Particle Swarm Optimization to Solving the Economic Dispatch Considering the Generator Constraints. IEEE Transactions on Power Systems, 18(3), 1187-1195.

22. Sinha, N., Chakrabarti, R., \& Chattopadhyay, P. K. (2003). Evolutionary programming techniques for economic load dispatch. IEEE Transactions on Evolutionary Computation, 7(1), 83-94. 